\title{
A class of new endomorphisms with some properties
}

\author{
Huang Xurong ${ }^{a^{*}}$, Hai Jinke ${ }^{b}$ and Chang Xiufeng ${ }^{c}$
}

\author{
School of Mathematics and Statistics, Qingdao University Shandong 266071, P.R.China \\ a1376882875@qq.com, ${ }^{\text {b haijinke@qdu.edu.cn, }{ }^{c} 1018402779 @ q q . c o m}$
}

Keywords: endomorphism; generalized morphic; generalized morphic group

\begin{abstract}
In this paper, we give a class of new endomorphisms with some properties based on the concept of morphic endomorphisms, called generalized morphic. We discuss the connection between the generalized morphic and the structure of groups. It is proved that the quasi-cyclic group is a generalized morphic group, rather than a morphic group. At the same time, we give out some applications of generalized morphic.
\end{abstract}

\section{Introduction}

G. Ehrlich investigated unit regular endomorphisms, and revealed the closely connection between the morphic endomorphisms and unit regular endomorphisms in [1]. He showed that an endomorphism $\alpha$ of a module ${ }_{R} M$ is unit regular if and only if it is regular and $M / M^{\alpha}$ $\cong \operatorname{Ker}(\alpha)$. W. K. Nicholson and M. F. Yousif proposed the concept of morphic and it has been extensively studied in module and ring theory respectively in [2] and [3]. They also obtained many famous results. Y. Li, W. K. Nicholson and L. Zan, investigated the concept of morphic in the category of groups, and gave an important connection between morphic endomorphisms and the structure of groups in [4].

In this paper, on the basis of the concept of morphic, we give a new observation of morphic endomorphism, called generalized morphic. Note that a morphic endomorphism is a generalized morphic endomorphism. Conversely, we can prove the following main results:

Theorem A Let $G$ be a quasi-cyclic group. Then $G$ is a generalized morphic group, rather than a morphic group.

As an application of generalized morphic groups, we have:

Theorem B Let $G$ be a generalized morphic group. If $K \triangleleft G$ and $K \cong G$, then $K=G$.

If $G$ is a group, we write $\operatorname{End}(G)$ for the monoid of endomorphisms $\alpha: G \rightarrow G$, and we write $\operatorname{Aut}(G)$ for the group of automorphisms of $G$. Let $\alpha$ be a endomorphism of $G$, denote by $G^{\alpha}$ the image of $\alpha$, and denote by $\operatorname{Ker}(\alpha)$ the kernel of $\alpha$. As usual we write $N^{+}$for the set of positive integers. And the other symbols are standard that can refer to [5].

\section{Generalized morphic}

Definition 2.1. Let $G$ be a group, $\alpha \in \operatorname{End}(G)$. If $G^{\alpha} \triangleleft G$, then $\alpha$ is said to be a normal endomorphism of $G$.

Definition 2.2. Let $G$ be a group, $\alpha \in \operatorname{End}(G)$. Then $\alpha$ is called morphic if $G^{\alpha} \triangleleft G$ and $G / G^{\alpha} \cong \operatorname{Ker}(\alpha)$; A group $G$ is called morphic if every endomorphism $\alpha$ of $G$ for which $G^{\alpha}$ is normal in $G$ satisfies $G / G^{\alpha} \cong \operatorname{Ker}(\alpha)$.

Proposition 2.3. Let $G$ be a group, the following are equivalent for $\alpha \in \operatorname{End}(G)$.

(1) $G^{\alpha} \triangleleft G$ and $G / G^{\alpha}$ can be embedded in $\operatorname{Ker}(\alpha)$.

(2) There exists $\beta \in \operatorname{End}(G)$ with $G^{\beta} \leq \operatorname{Ker}(\alpha)$ and $G^{\alpha}=\operatorname{Ker}(\beta)$.

(3) There exists $\beta \in \operatorname{End}(G)$ with $G^{\beta}$ can be embedded in $\operatorname{Ker}(\alpha)$ and $G^{\alpha}=\operatorname{Ker}(\beta)$.

Proof. $(1) \Rightarrow(2)$ By (1), then let $\sigma: G / G^{\alpha} \rightarrow \operatorname{Ker}(\alpha)$ is an injective homomorphism, define 
$\beta: G \rightarrow G$ by $g^{\beta}=\left(g G^{\alpha}\right)^{\sigma}$. It is clear that $\left(g G^{\alpha}\right)^{\sigma} \in G$. For arbitrary $g_{1}, g_{2} \in G$, if $g_{1}=g_{2}$, $g_{1} g_{2}^{-1}=1 \in G$. Therefore $g_{1} G^{\alpha}=g_{2} G^{\alpha}$. Since $\sigma$ is an injective homomorphism, $\left(g_{1} G^{\alpha}\right)^{\sigma}=\left(g_{2} G^{\alpha}\right)^{\sigma}$. Hence $\beta$ is well defined. Moreover, since $\left(g_{1} g_{2}\right)^{\beta}=\left(g_{1} g_{2} G^{\alpha}\right)^{\sigma}=g_{1}^{\beta} g_{2}^{\beta}, \beta \in \operatorname{End}(G)$. Hence $G^{\beta}=\left(G / G^{\alpha}\right)^{\sigma} \leq \operatorname{Ker}(\alpha)$ and $\operatorname{Ker}(\beta)=\left\{g \in G \mid\left(g G^{\alpha}\right)^{\sigma}=1\right\}=\left\{g \in G \mid g G^{\alpha}=G^{\alpha}\right\}=G^{\alpha}$.

$(2) \Rightarrow(3)$ This is obvious.

(3) $\Rightarrow(1)$ Given $\beta$ as in (3), then $G^{\alpha}=\operatorname{Ker}(\beta) \triangleleft G$ and $G / G^{\alpha}=G / \operatorname{Ker}(\beta) \cong G^{\beta}$. By (3),

$G / G^{\alpha}$ can be embedded in $\operatorname{Ker}(\alpha)$.

Definition 2.4. Let $G$ be a group, $\alpha \in \operatorname{End}(G)$. Then $\alpha$ is called generalized morphic if $G^{\alpha} \triangleleft G$ and $G / G^{\alpha}$ can be embedded in $\operatorname{Ker}(\alpha)$; A group $G$ is called generalized morphic if every endomorphism $\alpha$ of $G$ for which $G^{\alpha}$ is normal in $G$ satisfies $G / G^{\alpha}$ can be embedded in $\operatorname{Ker}(\alpha)$.

Note that morphic is generalized morphic. Conversely, we can prove the following result.

Definition 2.5. Let $C$ be the field of complex numbers and $p$ be an arbitrary prime where $C^{\times}$is the group of units of $C$. Write $C_{p^{\infty}}=\left\{z \in C^{\times} \mid\right.$there is a positive integer $n$ such that $\left.z^{p^{n}}=1\right\}$. Then the group $C_{p^{\infty}}$ is called quasi-cyclic groups.

Theorem 2.6. Let $G=C_{p^{\infty}}$ be a quasi-cyclic group. Then

(1) Every proper subgroup of $G$ is a cyclic group of order $p^{m}$. Write $C_{p^{m}}=\left\{z \in C^{\times} \mid z^{p^{m}}=1\right\}$, where $m$ is a non-negative integer.

(2) $\quad G / C_{p^{m}} \cong G, \quad m \geq 2$.

(3) There exists some $\alpha \in \operatorname{End}(G)$ such that $G^{\alpha} \triangleleft G$ is generalized morphic, but it is not morphic.

Proof. Suppose that $J$ is a proper subgroup of $G$, by the well ordering principle for the positive integers, we can let $m=\min \left\{n \in N^{+} \mid z^{p^{n}}=1\right.$, for arbitrary $\left.z \in J\right\}$. Write $C_{p^{m}}=\left\{z \in C^{\times} \mid z^{p^{m}}=1\right\}$. It is clear that $J=C_{p^{m}}$, i.e., every proper subgroup of $G$ is a cyclic group of order $p^{m}$. Write $C_{p^{m}}=\left\{z \in C^{\times} \mid z^{p^{m}}=1\right\}$, where $m$ is a non-negative integer.

Next, we shows that $G / C_{p^{m}} \cong G$. Let $\varphi: G / C_{p^{m}} \rightarrow G$ by $\left(z C_{p^{m}}\right)^{\varphi}=z^{p^{m}}, m \geq 2$. Let $z C_{p^{m}} \in G / C_{p^{m}}, z \in G$. Since $G$ is a group, $z^{p^{m}} \in G$. For every $z_{1}^{p^{m}}, z_{2}{ }^{p^{m}} \in G$, if $z_{1}^{p^{m}}=z_{2}^{p^{m}}$, which implies that $z_{1}^{-1} z_{2} \in C_{p^{m}}$. Therefore $\left(z_{1}^{-1} z_{2}\right)^{p^{m}}=1$, it follows that $z_{1}^{p^{m}}=z_{2}^{p^{m}}$, hence $\varphi$ is well defined. Since $\left(z_{1} C_{p^{m}} z_{2} C_{p^{m}}\right)^{\varphi}=\left(z_{1} z_{2}\right)^{p^{m}}=\left(z_{1} C_{p^{m}}\right)^{\varphi}\left(z_{2} C_{p^{m}}\right)^{\varphi}$, $\varphi$ is a homomorphism. Suppose that $z_{1}^{p^{m}}=z_{2} p^{p^{m}}$, then $\left(z_{1}^{p^{m}}\right)^{-1} z_{2}^{p^{m}}=1$. It obvious that $z_{1}^{-1} z_{2} \in C_{p^{m}}$, which implies that $z_{1} C_{p^{m}}=z_{2} C_{p^{m}}$, thus $\varphi$ is injective. For an arbitrary $z \in G$, there exists positive integer $i$, such that $z^{p^{i}}=1$. There also exists $x \in C^{\times}$such that $x^{p^{m}}=z$, which implies that $\left(x^{p^{m}}\right)^{p^{i}}=1$. Hence $x \in G$, it follows that $x^{\varphi}=x^{p^{m}}=z$, thus $\varphi$ is surjective. Moreover, $\varphi$ is isomorphic, that is, $G / C_{p^{m}} \cong G$.

Let $\gamma: G \rightarrow G / C_{p^{m}}$ be the natural homomorphism. Write $\alpha=\gamma \varphi$, given $\varphi$ as in (2). Then $\alpha: G \rightarrow G$ is an surjective homomorphism and $G^{\alpha}=G \triangleleft G$, thus $\alpha$ is a normal endomorphism and satisfies $\operatorname{Ker}(\alpha)=\left\{z \in G \mid z^{p^{m}}=1\right\}=C_{p^{m}} \neq 1$ and $G / G^{\alpha} \cong G / G=1$. Hence $G / G^{\alpha}$ can be embedded in $\operatorname{Ker}(\alpha)$, it follows that $\alpha$ is generalized morphic; Since $G / G^{\alpha}$ is not isomorphic to $\operatorname{Ker}(\alpha), \alpha$ is not generalized morphic. 
Theorem 2.7. Let $G=C_{p^{\infty}}$ be a quasi-cyclic group. Then $G$ is a generalized morphic group, but it is not a morphic group.

Proof. For any $\alpha \in \operatorname{End}(G)$, it is obvious that $G^{\alpha} \triangleleft G$. Suppose that $G^{\alpha}$ is proper normal in $G$, then there exists non-negative integer m such that $G^{\alpha}=\mathrm{C}_{p^{m}}$. It follows from the fundamental theorem on homomorphisms that $\operatorname{Ker}(\alpha)=G$. Hence $\alpha$ is the trivial endomorphism. It is clear that $G / G^{\alpha} \cong \operatorname{Ker}(\alpha)$, which implies that $\alpha$ is morphic and is also generalized morphic. Assume that $G^{\alpha}=1$, it follows from the fundamental theorem on homomorphisms that $\operatorname{Ker}(\alpha)=G$. Hence $\alpha$ is the trivial endomorphism which implies that $\alpha$ is morphic and is also generalized morphic. Suppose that $G^{\alpha}=G$, by the fundamental theorem on homomorphisms and theorem 1.6 (2), $\operatorname{Ker}(\alpha)=C_{p^{m}} \neq 1$ or $\operatorname{Ker}(\alpha)=1$. If $\operatorname{Ker}(\alpha)=1$, then $G / G^{\alpha} \cong \operatorname{Ker}(\alpha)$, hence $\alpha$ is morphic and is also generalized morphic. If $\operatorname{Ker}(\alpha)=C_{p^{m}} \neq 1$, then $G / G^{\alpha}$ can be embedded in $\operatorname{Ker}(\alpha)$. Therefore $\alpha$ is generalized morphic. However $G / G^{\alpha}$ is not isomorphic to $\operatorname{Ker}(\alpha)$, hence $\alpha$ is not generalized morphic. Therefore we conclude that $G$ is a generalized morphic group, rather than a morphic group.

Normal endomorphisms is not necessarily generalized morphic.

Example 2.8. Let $G$ denote the group $C_{2} \times C_{4}$. Then there exists $\alpha \in \operatorname{End}(\mathrm{G})$ such that $G^{\alpha} \triangleleft G$, but $\alpha$ is not generalized morphic.

Proof. Write $C_{2}=\langle a\rangle$ and $C_{4}=\langle b\rangle$. Define $\alpha: C_{2} \rightarrow C_{4}$ by $\left(a^{k}\right)^{\alpha}=b^{2 k}$, where $k \in N^{+}$. It is clear that $\alpha$ is a homomorphism. Define $\eta: G \rightarrow G$ by $(x, y)^{\eta}=\left(1, x^{\alpha}\right)$, then $\eta \in E n d(G)$ and $G^{\eta} \cong 1 \times C_{2} \triangleleft G$, it follows that $\eta$ is a normal endomorphism. By[5], we have $G / G^{\eta} \cong\left(C_{2} \times C_{4}\right) /\left(1 \times C_{2}\right) \cong C_{2} \times C_{2}, \operatorname{Ker}(\eta)=\left\{(x, y) \in G \mid(x, y)^{\eta}=1\right\}=\left\{(x, y) \in G \mid x^{\alpha}=1\right\}=1 \times C_{4}$. Therefore $G / G^{\eta}$ cannot be embedded in $\operatorname{Ker}(\eta)$, which implies that $\alpha$ is not generalized morphic.

\section{The application of generalized morphic}

Proposition 3.1. Let $\alpha \in \operatorname{End}(G)$ be generalized morphic. If $\alpha$ is one-to-one, then it is onto.

Proof. Suppose that $\alpha \in \operatorname{End}(G)$ is generalized morphic, then $G / G^{\alpha}$ can be embedded in $\operatorname{Ker}(\alpha)$. Since $\alpha$ is one-to-one, $G / G^{\alpha}$ can be embedded in $\operatorname{Ker}(\alpha)=1$, it follows that $G / G^{\alpha}=1$. Thus $G=G^{\alpha}$, which implies that $\alpha$ is onto. In particular, $\alpha \in \operatorname{Aut}(G)$.

Proposition 3.2. ${ }^{[4]}$ If $\alpha \in \operatorname{End}(G)$ is generalized morphic, so are $\alpha \rho$ and $\rho \alpha$ for every automorphism $\rho$ of $G$.

Corollary 3.3. Let $\alpha, \beta$ are generalized morphic. If $\alpha$ is one-to-one, $\beta$ is onto, then $\alpha \beta$, $\beta \alpha$ are generalized morphic.

Example 3.4. Let $G$ be a finite group, $K \triangleleft G$. If $K \cong G$, then $K=G$. Note that the result would fail if $G$ be an infinite group.

Proof. Write $G=Z^{+}$and $K=2 Z^{+}$, then $2 Z^{+} \triangleleft Z^{+}$. Let $\varphi: 2 Z^{+} \rightarrow Z^{+}$by $(2 n)^{\varphi}=n$. It clear that $\varphi$ is isomorphic, which implies that $2 Z^{+} \cong Z^{+}$but $2 Z^{+} \neq Z^{+}$.

Theorem 3.5. Let $G$ be generalized morphic, If $K \triangleleft G$ and $K \cong G$, then $K=G$.

Proof. Since $K \cong G$, let $\rho: G \rightarrow K$ be an isomorphism, define $\alpha: G \rightarrow G$ by $g^{\alpha}=g^{\rho}$. Certainly $\alpha \in \operatorname{End}(G)$. We have $\operatorname{Ker}(\alpha)=\left\{g \in G \mid g^{\rho}=1\right\}=1$ and $G^{\alpha}=G^{\rho}=K \triangleleft G$. Since $G$ is generalized morphic, $G / G^{\alpha}=G / K$ can be embedded in $\operatorname{Ker}(\alpha)$, it follows that $G / K=1$, that is, $G=K$. 
Theorem 3.6. Let $G_{1}, G_{2}$ be two isomorphic groups. If $G_{1}$ is generalized morphic, then $G_{2}$ is also generalized morphic.

Proof. Let $\varphi: G_{1} \rightarrow G_{2}$ be an isomorphism and let $\beta \in \operatorname{End}(G)$ such that $G_{2}{ }^{\beta} \triangleleft G_{2}$. It obvious that $\varphi \beta \varphi^{-1} \in \operatorname{End}\left(G_{1}\right)$. Since $G_{1}$ is a generalized morphic group, $G_{1}^{\varphi \beta \varphi^{-1}}=\left(G_{2}^{\beta}\right)^{\varphi^{-1}} \triangleleft G_{1}$ and $G_{1} / G_{1}^{\varphi \beta \varphi^{-1}}$ can be embedded in $\operatorname{Ker}\left(\varphi \beta \varphi^{-1}\right)$. Let $\psi: G_{1} \rightarrow G_{2} / G_{2}{ }^{\beta}$ by $x^{\psi}=x^{\varphi} G_{2}{ }^{\beta}$. For all $x_{1}, x_{2} \in G_{1},\left(x_{1} x_{2}\right)^{\psi}=\left(x_{1} x_{2}\right)^{\varphi} G_{2}{ }^{\beta}=x_{1}^{\varphi} x_{2}^{\varphi} G_{2}{ }^{\beta}=\left(x_{1}\right)^{\psi}\left(x_{2}\right)^{\psi}$, hence $\psi$ is a homomorphism. For any $x G_{2}{ }^{\beta} \in G_{2} / G_{2}^{\beta}, x \in G_{2}$, because $\varphi$ be an isomorphism, there exists $y \in G_{1}$ such that $y^{\varphi}=x$. Hence $y^{\psi}=x G_{2}^{\beta}$, which implies that $\psi$ is a surjective homomorphism. Now

$$
\operatorname{Ker}(\psi)=\left\{x \in G_{1} \mid x^{\psi}=1\right\}=\left\{x \in G_{1} \mid x^{\varphi} G_{2}^{\beta}=G_{2}^{\beta}\right\}=\left\{x \in G_{1} \mid x \in\left(G_{2}^{\beta}\right)^{\varphi^{-1}}\right\}=G_{1}^{\varphi \beta \varphi^{-1}}
$$

then $G_{1} / \operatorname{Ker}(\psi) \cong G_{1} / G_{1}^{\varphi \beta \varphi^{-1}} \cong G_{2} / G_{2}^{\beta}$.

Define $\theta: \operatorname{Ker}\left(\varphi \beta \varphi^{-1}\right) \rightarrow \operatorname{Ker}(\beta)$ by $y^{\theta}=y^{\varphi}$. For all $y \in \operatorname{Ker}\left(\varphi \beta \varphi^{-1}\right), \quad y^{\varphi \beta \varphi^{-1}}=1$. Hence $y^{\varphi \beta} \in \operatorname{Ker}\left(\varphi^{-1}\right)=1$, which implies that $y^{\varphi} \in \operatorname{Ker}(\beta)$, that is, $\theta$ is well defined. For arbitrary $y_{1}, y_{2} \in \operatorname{Ker}\left(\varphi \beta \varphi^{-1}\right), \quad\left(y_{1} y_{2}\right)^{\theta}=\left(y_{1} y_{2}\right)^{\varphi}=y_{1}^{\varphi} y_{2}^{\varphi}=y_{1}^{\theta} y_{2}^{\theta}$. Hence $\theta$ is a homomorphism. Because $\operatorname{Ker}(\theta)=1$, so that $\theta$ is injective. For any $y \in \operatorname{Ker}(\beta) \leq G_{2}$, there exists $x \in G$ such that $x^{\varphi}=y$. Since $x^{\varphi \beta \varphi^{-1}}=\left(\left(x^{\varphi}\right)^{\beta}\right)^{\varphi^{-1}}=\left(y^{\beta}\right)^{\varphi^{-1}}=1, x \in \operatorname{Ker}\left(\varphi \beta \varphi^{-1}\right)$. It follows that $\theta$ is surjective, therefore $\theta$ is isomorphic, that is, $\operatorname{Ker}\left(\varphi \beta \varphi^{-1}\right) \cong \operatorname{Ker}(\beta)$. Hence $G_{2} / G_{2}^{\beta}$ can be embedded in $\operatorname{Ker}\left(\varphi \beta \varphi^{-1}\right)$, so that $G_{2}$ is generalized morphic.

\section{References}

[1] G. Ehrlich, Unites and one-sided units in regular ring, Trans. Amer. Math. Soc. 216 (1976) 81-90.

[2] W.K. Nicholson, E. Sánchez Campos, Ring with the dual of the isomorphism theorem, J. Algebra 271 (2004) 391-406.

[3] W.K. Nicholson, E. Sánchez Campos, Morphic modules, Comm. Algebra 33 (2005) 2629-264.

[4] Y. Li, W.K. Nicholson, L. Zan, Morphic groups, J. Pure App. Algebra 214 (2010) 1827-1834.

[5] J.S. Rose, A course on group theory, Cambridge University Press, Cambridge, 1978.

[6] Q. Wang, K. Long, L. Feng, The quasi-morphic property of group, Bulletin of Iranian Mathematical Society 39 (2013) 175-185.

[7] Rabah Kellil, Some propreties on morphic groups, Pure Mathematical Sciences 2 (2013) 55-68. 